\title{
On the Convergence Rate of Newton Interior-Point Methods in the Absence of Strict Complementarity
}

\author{
A. S. El-Bakry, R. A. Tapia ${ }^{2}$ and Y. Zhang ${ }^{3}$
}

May, 1995

\begin{abstract}
In the absence of strict complementarity, Monteiro and Wright [7] proved that the convergence rate for a class of Newton interior-point methods for linear complementarity problems is at best linear. They also established an upper bound of $1 / 4$ for the $Q_{1}$-factor of the duality gap sequence when the steplengths converge to one. In the current paper, we prove that the $Q_{1}$ factor of the duality gap sequence is exactly $1 / 4$. In addition, the convergence of the Tapia indicators is also discussed.
\end{abstract}

Keywords: Interior-point methods, linear convergence, strict complementarity, $Q_{1}$-factors, the Tapia indicators.

Abbreviated Title: Convergence in the Absence of Strict Complementarity

AMS(MOS) subject classifications: $65 \mathrm{~K}, 49 \mathrm{M}, 90 \mathrm{C}$

\section{Introduction}

In this paper we consider the monotone linear complementarity problem: find $(x, y) \in \mathbb{R}^{n} \times \mathbb{R}^{n}$ such that

$$
y=M x+q, \quad(x, y) \geq 0, \quad x^{T} y=0,
$$

\footnotetext{
${ }^{1}$ Department of Mathematics, Faculty of Science, Alexandria University, Alexandria, Egypt and the Center for Research on Parallel Computations, Rice University, Houston, Texas, 77521-1892 (elbakry@rice.edu). This author was supported in part by NSF Coop. Agr. No. CCR-8809615 and AFOSR 89-0363 and the REDI Foundation

${ }^{2}$ Department of Computational and Applied Mathematics and the Center for Research on Parallel Computations, Rice University, Houston, Texas, 77521-1892 (rat@rice.edu). This author was supported in part by NSF Coop. Agr. No. CCR-8809615, AFOSR 89-0363, DOE DEFG05-86ER25017 and ARO 9DAAL03-90-G-0093

${ }^{3}$ Department of Mathematics and Statistics, University of Maryland Baltimore County, Baltimore, MD 21228, and visiting member of the Center for Research on Parallel Computations, Rice University, Houston, Texas, $77251-1892$ (yzhang@math.umbc.edu). This author was supported in part by DOE DE-FG02-93ER25171
} 
where $q \in \mathbb{R}^{n}$, and $M \in \mathbb{R}^{n \times n}$ is positive semi-definite. We often write (1.1) in the equivalent form

$$
F(x, y) \equiv\left(\begin{array}{c}
M x-y+q \\
X Y e
\end{array}\right)=0, \quad(x, y) \geq 0,
$$

where $X=\operatorname{diag}(x), Y=\operatorname{diag}(y)$ and $e$ is the vector of all ones in $\mathbb{R}^{n}$. Let $\mathcal{S}$ denote the solution set of problem (1.1). We are interested in the following three subsets of the index set $\{1, \ldots, n\}$

$$
\begin{aligned}
\mathcal{B} & =\left\{i: x_{i}^{*}>0 \text { for at least one }\left(x^{*}, y^{*}\right) \in \mathcal{S}\right\}, \\
\mathcal{N} & =\left\{i: y_{i}^{*}>0 \text { for at least one }\left(x^{*}, y^{*}\right) \in \mathcal{S}\right\}, \\
\mathcal{J} & =\left\{i: x_{i}^{*}=y_{i}^{*}=0 \text { for all }\left(x^{*}, y^{*}\right) \in \mathcal{S}\right\} .
\end{aligned}
$$

It is well known that $\mathcal{B}, \mathcal{N}$, and $\mathcal{J}$ form a partition of $\{1, \ldots, n\}$. A solution pair $(x, y)$ is said to satisfy strict complementarity if in addition to complementarity $X Y e=0$, it satisfies $x+y>0$. In this case, $\mathcal{J}=\emptyset$ and problem (1.1) is said to be nondegenerate. Otherwise, it is said to be a degenerate monotone complementarity problem.

Primal-dual Newton interior-point methods have proved to be very effective methods for linear programming problems. This success led researchers to extend the primal-dual interior-point algorithmic framework to more general problems. One of the key ingredients that contribute to the success of primal-dual methods is their superlinear convergence rate. This topic was first studied, for these methods, by Zhang, Tapia, and Dennis [10]. To prove $Q$-superlinear convergence, strict complementarity is assumed in most theoretical results that followed the work of Zhang, Tapia and Dennis. For linear programming problems, the existence of a strict complementary solution was established by Goldman and Tucker [3]. However, for monotone linear complementarity problems such solutions may not exist.

The question as to whether the assumption of strict complementarity is necessary for superlinear convergence was answered in the affirmative by Monteiro and Wright [7] for a generic class of interiorpoint algorithms that will be defined in the next section. They considered the degenerate monotone linear complementarity problem (1.1) and proved that for this class of methods the convergence rate of the duality gap $\left(x^{T} y\right)$ is at best linear with $Q_{1}$-factor less than or equal to $1 / 4$. This result requires that the steplengths converge to one, which seems to be rather difficult to enforce for degenerate problems. The question arises whether fast linear convergence with $Q_{1}$-factor less than $1 / 4$ is attainable.

In this paper, we prove that the $Q_{1}$-factor of the duality gap sequence is exactly $1 / 4$. This result implies that for the considered class of algorithms fast linear convergence cannot be attained. It lends support to the recent activity in designing algorithms that achieve superlinear convergence in 
the absence of strict complementarity, see Mizuno [6] and Potra and Sheng [9]. We will also briefly discuss the convergence of the Tapia indicators in the absence of strict complementarity.

This paper is organized as follows. In Section 2 we introduce a generic interior-point algorithmic framework. In Section 3 we establish our main result concerning the $Q_{1}$-factor of the duality gap sequence. In Section 4, we discuss questions concerning the convergence and convergence rate of the Tapia indicators in the absence of strict complementarity. In Section 5 we give a numerical example. We conclude the paper in Section 6 .

\section{A Generic Interior-Point Method}

The generic interior-point method under consideration in this paper is essentially a damped and perturbed Newton method applied to the system of equations and inequalities (1.2). It is a feasible interior-point method with the following structure. Recall that by a feasible point we mean a point which satisfies the linear relationships in (1.2) and by a strictly feasible point we mean the linear inequality is strictly satisfied.

\section{Algorithm 2.1}

Given a strictly feasible starting point $\left(x^{0}, y^{0}\right)$. For $k=0,1, \ldots$, do

1. Choose $\sigma^{k} \in[0,1)$ and set $\mu^{k}=\sigma^{k} \frac{\left(x^{k}\right)^{T} y^{k}}{n}$.

2. Solve the following system for $\left(\Delta x^{k}, \Delta y^{k}\right)$ :

$$
F^{\prime}\left(x^{k}, y^{k}\right)(\Delta x, \Delta y)=-F\left(x^{k}, y^{k}\right)+\mu^{k}\left(\begin{array}{l}
0 \\
e
\end{array}\right) .
$$

3. Choose $\tau^{k} \in(0,1]$ and set the steplength $\alpha^{k}=\min \left(1, \tau^{k} \hat{\alpha}^{k}\right)$ where

$$
\hat{\alpha}^{k}=\frac{-1}{\min \left(\left(X^{k}\right)^{-1} \Delta x^{k},\left(Y^{k}\right)^{-1} \Delta y^{k},-\frac{1}{2}\right)} .
$$

4. Form the new iterate

$$
\left(x^{k+1}, y^{k+1}\right)=\left(x^{k}, y^{k}\right)+\alpha^{k}\left(\Delta x^{k}, \Delta y^{k}\right) .
$$

The perturbed Newton system (2.1) can be written

$$
\left(\begin{array}{cc}
M & -I \\
Y^{k} & X^{k}
\end{array}\right)\left(\begin{array}{c}
\Delta x \\
\Delta y
\end{array}\right)=-\left(\begin{array}{c}
0 \\
X^{k} Y^{k} e-\mu^{k} e
\end{array}\right) .
$$

Throughout this paper we will assume that the following conditions hold for the iteration sequence $\left\{\left(x^{k}, y^{k}\right)\right\}$ generated by Algorithm 2.1. 
(C1) $\left(x^{k}\right)^{T} y^{k} \rightarrow 0$

(C2) $x_{i}^{k} y_{i}^{k} \geq \gamma\left(x^{k}\right)^{T} y^{k} / n$ for $i=1, \ldots, n$ and some positive constant $\gamma$.

Condition $\mathrm{C} 1$ implies that the sequence $\left\{\left(x^{k}, y^{k}\right)\right\}$ converges to the solution set of problem (1.1). Condition $\mathrm{C} 2$ is a widely used, practical centrality condition.

The following result, Lemma 4.5 of Monteiro and Wright [7], is essential in our analysis.

\section{Lemma 2.1 (Monteiro and Wright)}

Let the sequence $\left\{\left(x^{k}, y^{k}\right)\right\}$ be generated by Algorithm 2.1 and satisfy Conditions C1-C2. If $\mathcal{J} \neq \emptyset$ and $\sigma^{k} \rightarrow 0$, then for all $i \in \mathcal{J}$

$$
\lim _{k \rightarrow \infty} \frac{\Delta x_{i}^{k}}{x_{i}^{k}}=\lim _{k \rightarrow \infty} \frac{\Delta y_{i}^{k}}{y_{i}^{k}}=-\frac{1}{2}
$$

\section{The $Q_{1}$-factor of the Gap Sequence}

In this section, we establish our main result for the $Q_{1}$-factor of the gap sequence $\left\{\left(x^{k}\right)^{T} y^{k}\right\}$. We prove that if the steplength sequence $\left\{\alpha^{k}\right\}$ converges to one, then the $Q_{1}$-factor of the duality gap $\left\{\left(x^{k}\right)^{T} y^{k}\right\}$ is exactly $1 / 4$. In Section 5 , we will provide numerical experiments that validate our theory.

We first establish a lemma on the $Q_{1}$-factor of a sequence whose general term is the sum of terms of a finite number of individual sequences with known $Q_{1}$-factors. We establish bounds on the $Q_{1}$-factor of the summation sequence in terms of the $Q_{1}$ factors of the individual sequences. This result is crucial to our subsequent analysis and seems to be of interest in its own right.

For any sequence $\left\{a^{k}\right\}$ of positive real numbers, we define the set

$$
Q_{1}^{*}\left(\left\{a^{k}\right\}\right)=\left\{z: z \text { is a limit point of } \frac{a^{k+1}}{a^{k}}\right\},
$$

i.e. the derived set of the sequence $\left\{a^{k+1} / a^{k}\right\}$. Recall that if $\left\{a^{k}\right\}$ converges to zero, then the $Q_{1}$-factor of $\left\{a^{k}\right\}$ is the largest element of $Q_{1}^{*}\left(\left\{a^{k}\right\}\right)$, see Ortega and Rheinboldt [8].

Lemma 3.1 Let $\left\{a_{1}^{k}\right\},\left\{a_{2}^{k}\right\}, \ldots,\left\{a_{p}^{k}\right\}$ be sequences of positive real numbers converging to zero with $Q_{1}$-factors $q_{1}, q_{2}, \ldots, q_{p}$, ordered so that

$$
q_{1} \leq q_{2} \leq \ldots \leq q_{p}
$$

Then the sequence $\left\{\sum_{j=1}^{p} a_{j}^{k}\right\}$ converges to zero with $Q_{1}$-factor $q_{s}$ satisfying

$$
q_{1} \leq q_{s} \leq q_{p}
$$


Moreover, if $Q_{1}^{*}\left(\left\{a_{p}^{k}\right\}\right)$ does not contain zero, then $q_{s}$ is greater than or equal to the smallest nonzero $Q_{1}$-factor among $q_{1}, q_{2}, \ldots, q_{p}$.

Proof: Consider the sequence $\left\{\theta^{k}\right\}$, where $\theta^{k}=\sum_{j=1}^{p} a_{j}^{k}$ and let

$$
\pi^{k}=\prod_{j=1}^{p} a_{j}^{k} \text { and } \pi_{j}^{k}=\prod_{i \neq j, 1 \leq i \leq p} a_{i}^{k} .
$$

Consider the $Q_{1}$-factor of the sequence $\left\{\theta^{k}\right\}$ :

$$
q_{s}=Q_{1}\left(\left\{\theta^{k}\right\}\right)=\limsup _{k \rightarrow \infty} \frac{\sum_{j=1}^{p} a_{j}^{k+1}}{\sum_{j=1}^{p} a_{j}^{k}} .
$$

Observe that

$$
\frac{\sum_{j=1}^{p} a_{j}^{k+1}}{\sum_{j=1}^{p} a_{j}^{k}}=\frac{\sum_{j=1}^{p} a_{j}^{k+1} / \pi^{k}}{\sum_{j=1}^{p} a_{j}^{k} / \pi^{k}}=\left[\sum_{j=1}^{p} \frac{a_{j}^{k+1}}{a_{j}^{k}} \frac{1}{\pi_{j}^{k}}\right] /\left[\sum_{j=1}^{p} \frac{1}{\pi_{j}^{k}}\right] .
$$

Hence,

$$
\min _{1 \leq j \leq p} \frac{a_{j}^{k+1}}{a_{j}^{k}} \leq \frac{\sum_{j=1}^{p} a_{j}^{k+1}}{\sum_{j=1}^{p} a_{j}^{k}} \leq \max _{1 \leq j \leq p} \frac{a_{j}^{k+1}}{a_{j}^{k}} .
$$

Taking the limit superior of the above inequality gives inequality (3.2).

To prove the second part of the lemma, assume that $Q_{1}^{*}\left(\left\{a_{p}^{k}\right\}\right)$ does not contain zero. This implies that the sequence $\left\{a_{p}^{k+1} / a_{p}^{k}\right\}$ is bounded away from zero.

Without loss of generality, assume that

$$
0=q_{1}=\ldots=q_{r-1}<q_{r} \leq \cdots \leq q_{p} .
$$

Hence we need to prove that $Q_{1}\left(\left\{\theta^{k}\right\}\right) \geq q_{r}$.

Let us write $\theta^{k}=\theta_{0}^{k}+\theta_{+}^{k}$, where

$$
\theta_{0}^{k}=\sum_{j=1}^{r-1} a_{j}^{k} \quad \text { and } \quad \theta_{+}^{k}=\sum_{j=r}^{p} a_{j}^{k} .
$$

It is clear from (3.1) that $\left\{\theta_{0}^{k}\right\}$ converges to zero $Q$-superlinearly, i.e.,

$$
\lim _{k \rightarrow \infty} \frac{\theta_{0}^{k+1}}{\theta_{0}^{k}}=0
$$

Furthermore, we have

$$
0=Q_{1}\left(\left\{\theta_{0}^{k}\right\}\right) \leq Q_{1}\left(\left\{\theta^{k}\right\}\right) \leq Q_{1}\left(\left\{\theta_{+}^{k}\right\}\right) \quad \text { and } \quad q_{r} \leq Q_{1}\left(\left\{\theta_{+}^{k}\right\}\right) \leq q_{p}
$$


First we prove that

$$
\lim _{k \rightarrow \infty} \frac{\theta_{0}^{k}}{\theta_{+}^{k}}=0 .
$$

Since $\theta_{0}^{k} / \theta_{+}^{k} \leq \theta_{0}^{k} / a_{p}^{k}$, it suffices to show that $\theta_{0}^{k} / a_{p}^{k} \rightarrow 0$. Actually, this sequence, $\left\{\theta_{0}^{k} / a_{p}^{k}\right\}$, converges to zero $Q$-superlinearly. Consider the quotient

$$
\frac{\theta_{0}^{k+1} / a_{p}^{k+1}}{\theta_{0}^{k} / a_{p}^{k}}=\frac{\theta_{0}^{k+1} / \theta_{0}^{k}}{a_{p}^{k+1} / a_{p}^{k}} .
$$

Since the numerator converges to zero and the denominator is bounded away from zero. The limit of the quotient is zero. Hence, $\left\{\theta_{0}^{k} / a_{p}^{k}\right\}$ converges to zero $Q$-superlinearly.

Now we prove that

$$
Q_{1}\left(\left\{\theta^{k}\right\}\right)=Q_{1}\left(\left\{\theta_{+}^{k}\right\}\right)
$$

Toward this end, observe that

$$
\frac{\theta^{k+1}}{\theta^{k}}=\frac{\theta_{0}^{k+1}+\theta_{+}^{k+1}}{\theta_{0}^{k}+\theta_{+}^{k}} \geq \frac{\theta_{+}^{k+1} / \theta_{+}^{k}}{1+\theta_{0}^{k} / \theta_{+}^{k}}
$$

and that the denominator in the right-hand side converges to one. Taking the limit superior of both sides, we obtain

$$
Q_{1}\left(\left\{\theta^{k}\right\}\right) \geq Q_{1}\left(\left\{\theta_{+}^{k}\right\}\right)
$$

which, together with (3.3), completes the proof.

Now we give our main result concerning the $Q_{1}$-factor of the gap sequence $\left\{\left(x^{k}\right)^{T} y^{k}\right\}$.

Proposition 3.1 Let $\left\{\left(x^{k}, y^{k}\right)\right\}$ be generated by Algorithm 2.1 and satisfy Conditions C1-C2. If

1. $\mathcal{J} \neq \emptyset$,

2. the centering parameters $\sigma^{k}$ are chosen so that $\sigma^{k} \rightarrow 0$,

3. $\alpha^{k} \rightarrow 1$,

then the gap sequence $\left\{\left(x^{k}\right)^{T} y^{k}\right\}$ converges to zero Q-linearly with $Q_{1}$-factor

$$
Q_{1}\left(\left\{\left(x^{k}\right)^{T} y^{k}\right\}\right)=\frac{1}{4}
$$

Proof: First consider linearized complementarity, which is the second block of equations in (2.2)

$$
y_{i} \Delta x_{i}+x_{i} \Delta y_{i}=-x_{i} y_{i}+\sigma x^{T} y / n,
$$


i.e.,

$$
\frac{\Delta x_{i}^{k}}{x_{i}^{k}}+\frac{\Delta y_{i}^{k}}{y_{i}^{k}}=-1+\sigma^{k} \frac{\left(x^{k}\right)^{T} y^{k} / n}{x_{i}^{k} y_{i}^{k}} .
$$

Thus for all $i \in\{1, \ldots, n\}$, we have

$$
\frac{x_{i}^{k}+\alpha^{k} \Delta x_{i}^{k}}{x_{i}^{k}}+\frac{y_{i}^{k}+\alpha^{k} \Delta y_{i}^{k}}{y_{i}^{k}}=\left(2-\alpha^{k}\right)+\sigma^{k} \frac{\left(x^{k}\right)^{T} y^{k} / n}{x_{i}^{k} y_{i}^{k}} .
$$

It follows from condition $\mathrm{C} 2$ and $\sigma^{k} \rightarrow 0$ that the last term above tends to zero. Hence

$$
\lim _{k \rightarrow \infty} \frac{x_{i}^{k+1}}{x_{i}^{k}}+\frac{y_{i}^{k+1}}{y_{i}^{k}}=1 .
$$

It is well known that, under condition $\mathrm{C} 2,\left\{x_{i}^{k}\right\}$ is bounded away from zero for $i \in \mathcal{B}$ and $\left\{y_{i}^{k}\right\}$ is bounded away from zero for $i \in \mathcal{N}$ (Lemma 2.2 of Monteiro and Wright [7]). Moreover, from Lemma 4.4 of Monteiro and Wright [7] we have

$$
\Delta x_{i}^{k}=O\left(\left[\left(x^{k}\right)^{T} y^{k}\right]^{1 / 2}+\sigma^{k}\right), \quad i \in \mathcal{B},
$$

and

$$
\Delta y_{i}^{k}=O\left(\left[\left(x^{k}\right)^{T} y^{k}\right]^{1 / 2}+\sigma^{k}\right), \quad i \in \mathcal{N} .
$$

Since $\left(x^{k}\right)^{T} y^{k} \rightarrow 0$ and $\sigma^{k} \rightarrow 0$, we have $\Delta x_{i}^{k} / x_{i}^{k} \rightarrow 0$ for $i \in \mathcal{B}$. Hence

$$
\lim _{k \rightarrow \infty} \frac{x_{i}^{k+1}}{x_{i}^{k}}=1 \text { and } \lim _{k \rightarrow \infty} \frac{y_{i}^{k+1}}{y_{i}^{k}}=0, \text { for all } i \in \mathcal{B} .
$$

Similar arguments yield

$$
\lim _{k \rightarrow \infty} \frac{y_{i}^{k+1}}{y_{i}^{k}}=1 \text { and } \lim _{k \rightarrow \infty} \frac{x_{i}^{k+1}}{x_{i}^{k}}=0, \text { for all } i \in \mathcal{N} .
$$

Let us now consider the case of $i \in \mathcal{J}$. From Lemma 2.1 and the condition that $\alpha^{k} \rightarrow 1$ it follows that for $i \in \mathcal{J}$

$$
\lim _{k \rightarrow \infty} \frac{x_{i}^{k+1}}{x_{i}^{k}}=\lim _{k \rightarrow \infty} \frac{y_{i}^{k+1}}{y_{i}^{k}}=\frac{1}{2}, \text { for all } i \in \mathcal{J} .
$$

Now consider the gap sequence $\left\{\left(x^{k}\right)^{T} y^{k}\right\}$

$$
\left(x^{k}\right)^{T} y^{k}=\sum_{i=1}^{n} x_{i}^{k} y_{i}^{k}
$$

In the above we have shown that

$$
\lim _{k \rightarrow \infty} \frac{x_{i}^{k+1} y_{i}^{k+1}}{x_{i}^{k} y_{i}^{k}}=\left\{\begin{array}{c}
1 / 4, \text { for all } i \in \mathcal{J} \\
0, \text { otherwise }
\end{array}\right.
$$


Therefore from Lemma 3.1 we obtain

$$
\frac{1}{4} \leq \limsup _{k \rightarrow \infty} \frac{\left(x^{k+1}\right)^{T} y^{k+1}}{\left(x^{k}\right)^{T} y^{k}} \leq \frac{1}{4}
$$

which completes the proof.

\section{Convergence of the Tapia Indicators}

In this section, we discuss the convergence of the Tapia indicators in the absence of strict complementarity. Our result follows directly from Lemma 4.4 and Lemma 4.5 of Monteiro and Wright [7]. The Tapia indicators have already been used in the context of designing superlinearly convergent algorithms in the absence of strict complementarity (see Potra and Sheng [9]).

The primal and dual Tapia indicators have the form

$$
\frac{x_{i}^{k+1}}{x_{i}^{k}} \text { and } \frac{y_{i}^{k+1}}{y_{i}^{k}},
$$

respectively. A popular and direct variant of this indicator is given by

$$
\frac{\Delta x_{i}^{k}}{x_{i}^{k}} \text { and } \frac{\Delta y_{i}^{k}}{y_{i}^{k}} .
$$

The convergence and convergence rate of these indicators in the framework of interior-point methods for linear programming were investigated by El-Bakry [1] and El-Bakry, Tapia, and Zhang [2].

Monteiro and Wright [7] proved that in the absence of strict complementarity the step variant (4.1) of the Tapia indicators converges for pairs $\left(x_{i}, y_{i}\right)$ with $i \in \mathcal{J}$ (see Lemma 2.1 above). In the proof of Proposition 3.1 above, we see that the Tapia indicators for pairs $\left(x_{i}, y_{i}\right)$ with $i \in \mathcal{B}$ and $i \in \mathcal{N}$ converge to 1 and 0 , respectively, when $\sigma^{k} \rightarrow 0$ and $\alpha^{k} \rightarrow 1$. However, in our numerical experiments, we found it difficult to choose the algorithmic parameters so that both of these conditions are satisfied when $\mathcal{J}$ is nonempty. The same observation is also reported by Monteiro and Wright [7]. However, it is easy to see that if we define the primal (similarly for dual) Tapia indicators always using $\alpha^{k}=1$, i.e.,

$$
T_{p}^{k}=\frac{x_{i}^{k}+\Delta x_{i}^{k}}{x_{i}^{k}},
$$

then the same convergence results hold without the assumption that the steplength sequence $\left\{\alpha^{k}\right\}$ converges to one. The modification of these indicators that always takes $\alpha^{k}=1$ has been considered in other applications, see for example El-Bakry, Tapia, and Zhang [2]. For the sake of completeness, we summarize the convergence result for the above primal Tapia indicators in the next corollary. 
Corollary 4.1 Let $\left\{\left(x^{k}, y^{k}\right)\right\}$ be generated by Algorithm 2.1 and satisfy Conditions C1-C2. If $\mathcal{J}$ is not empty and the centering parameters $\sigma^{k}$ are chosen so that $\sigma^{k} \rightarrow 0$, then

$$
\lim _{k \rightarrow \infty} T_{p}^{k}= \begin{cases}1, & i \in \mathcal{B} \\ \frac{1}{2}, & i \in \mathcal{J} \\ 0, & i \in \mathcal{N}\end{cases}
$$

\section{A Numerical Example}

In this section, we present some preliminary numerical experiments using the generic interior-point algorithm. These experiments were performed on a Sun SPARCstation 10 Model 41 workstation running SunOS 4.1.3. Our code was written in MATLAB.

In our implementation, we used the following parameter choices

$$
\sigma^{k}=\min \left(0.2, \bar{C}\left(x^{k}\right)^{T} y^{k}\right) \text { and } \tau^{k}=\max \left(0.8,1-\bar{C}\left(x^{k}\right)^{T} y^{k}\right),
$$

where $\bar{C}=10^{2}$. The stopping criterion that we used is $\left\|F\left(x^{k}, y^{k}\right)\right\|_{2} \leq 10^{-8}$.

The test problem we used is a modification of Problem 32 in Hock and Schittkowski [5]. The modified problem has the form

$$
\begin{array}{ll}
\text { minimize } & \left(x_{1}+3 x_{2}+x_{3}\right)^{2}+4\left(x_{1}-x_{2}\right)^{2} \\
\text { subject to } & 1-x_{1}-x_{2}-x_{3}=0 \\
& 6 x_{2}+4 x_{3}-x_{1}-x_{4}-3=0 \\
& \left(x_{1}, x_{2}, x_{3}, x_{4}\right) \geq 0
\end{array}
$$

This is a convex quadratic programming problem and thus a special case of (1.1). The solution to this problem does not satisfy strict complementarity. Indeed the solution to this problem is $x^{*}=(0,0,1,1)$ and the Lagrange multiplier vector corresponding to the nonnegativity constraints is $y^{*}=(0,4,0,0)$. Note that both $x_{1}^{*}$ and $y_{1}^{*}$ are zero at the solution.

It should be mentioned that in our numerical experiment, the iterates are not feasible with respect to the linear equations $M x-y+q=0$. However, our numerical experiments demonstrate that even in this case both quotients

$$
\frac{\left\|F\left(x^{k+1}, y^{k+1}\right)\right\|}{\left\|F\left(x^{k}, y^{k}\right)\right\|} \text { and } \frac{\left(x^{k+1}\right)^{T} y^{k+1}}{\left(x^{k}\right)^{T} y^{k}}
$$

converge to $1 / 4$.

Figure 1 demonstrates the behavior of the above quotients in two different experiments. 
1. In the top plot, the centrality condition $\mathrm{C} 2$ is imposed with $\gamma=10^{-6}$. This caused the algorithm to take very small steps and severely slowed down the convergence. In this case, the steplength sequence $\left\{\alpha^{k}\right\}$ did not approach one and both quotients appeared to approach one, indicating possible sublinear convergence. Indeed, we had to take 100 iterations to reduce the residual to $1.769 \times 10^{-6}$. In our numerical experiments, we found out that it is rather difficult to design algorithms that enforce the centrality condition $\mathrm{C} 2$ and force the steplength sequence to converge to one at the same time.

2. In the bottom plot, the centrality condition is not enforced. The steplength sequence effectively approaches one and the algorithm demonstrates linear convergence with a $Q_{1}$-factor of $1 / 4$. This can be seen from the plot where both quotients approach $1 / 4$.
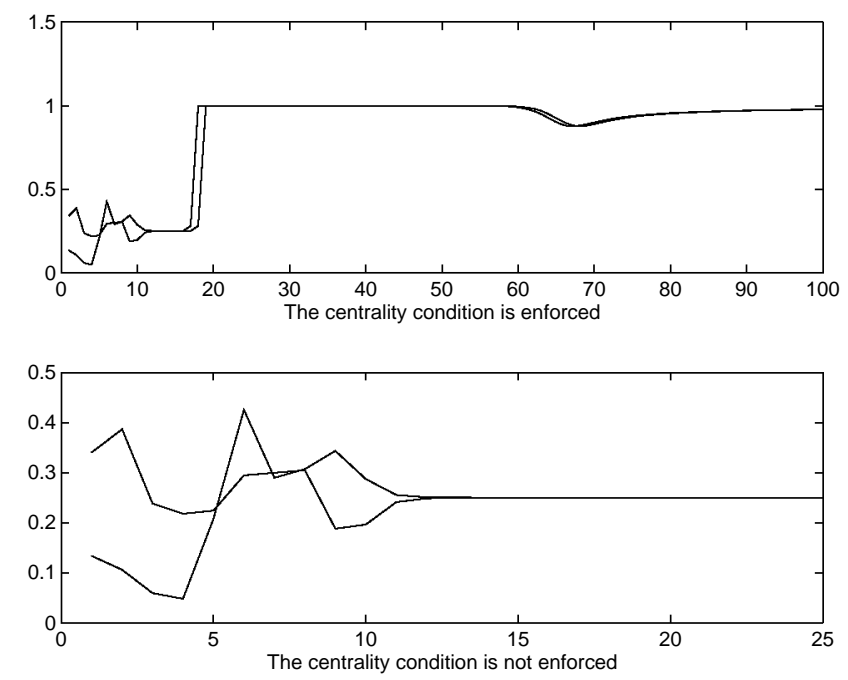

Figure 1: The behavior of residual and duality gap

It is worth mentioning that, when the centrality condition C2 is imposed, the primal Tapia indicators converge to the terminal values predicted by Corollary 4.1. This is demonstrated in Figure 2.

\section{Conclusion}

In the absence of strict complementarity, we established that, for a certain class of interior-point methods, the $Q_{1}$ factor of the duality gap is exactly $1 / 4$ when the steplength sequence converges to one. This result demonstrates that faster convergence cannot be attained by algorithms in the framework of the generic algorithm 2.1. It also lends support to the recent activity in designing fast 


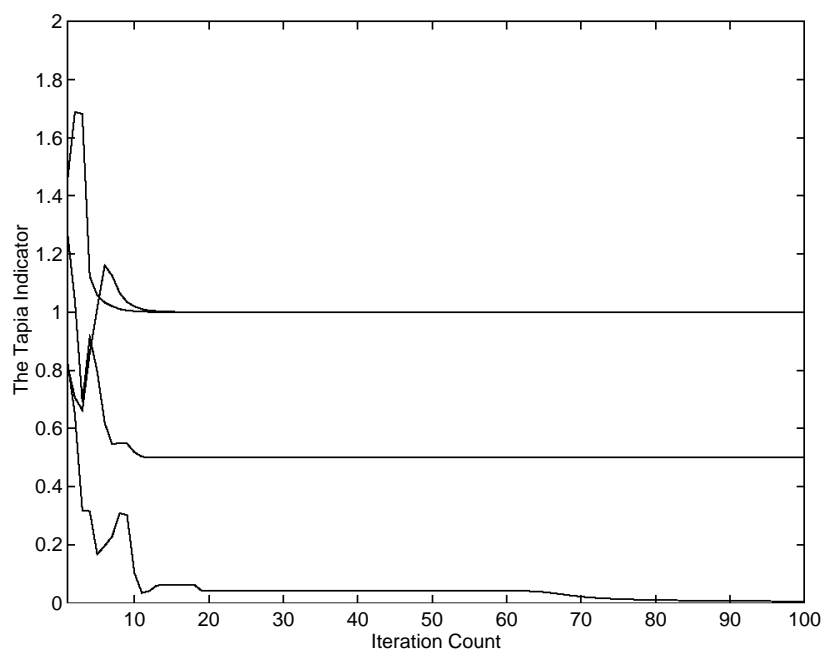

Figure 2: The behavior of the primal Tapia indicators

algorithms for degenerate problems, see Mizuno [6] and Potra and Sheng [9]. In these new algorithms the identification of variable pairs with indices in $\mathcal{J}$ is important. Corollary 3.1 provides a basis for the use of the Tapia indicators to identify such variables.

\section{References}

[1] A. S. El-Bakry. On the role of indicators in identifying zero variables in linear programming. PhD thesis, Department of Mathematical Sciences, Rice University, Houston, Texas 77251, 1991.

[2] A. S. El-Bakry, R. A. Tapia, and Y. Zhang. A study of indicators for identifying zero variables in interior-point methods. SIAM Review, 36:45-72, 1994.

[3] A. J. Goldman and A. W. Tucker. Theory of linear programming. In H. W. Kuhn and A. W. Tucker, editors, Linear inequalities and related systems, pages 53-97. Princeton University Press, 1956.

[4] O. Güler and Y. Ye. Convergence behavior of some interior-point algorithms. Math. Programming, Series A, 60:215-228, 1993.

[5] W. Hock and K. Schittkowski. Test Examples for Nonlinear Programming Codes. SpringerVerlag, New York, 1981. 
[6] S. Mizuno. A superlinearly convergent infeasible interior-point algorithm for geometric lcps without a strictly complementary solution. Technical Report 878, School of Operations Research and Industrial Engineering, Cornell University, 1994.

[7] R.C. Monteiro and S. Wright. Local convergence of interior-point algorithms for degenerate monotone lcp problems. Technical report, Mathematics and Computer Science Division, Argonne National Laboratory, 1993. MSC-P357-0493.

[8] J. M. Ortega and W. C. Rheinboldt. Iterative Solution of Nonlinear Equations in Several Variables. Academic Press, New York, 1970.

[9] F.A. Potra and R. Sheng. A superlinearly convergent infeasible interior-point algorithm for degenerate lcp. Technical Report 66, Department of Mathematics, Iowa University, Iowa 52242, 1995.

[10] Y. Zhang, R. A. Tapia, and J. E. Dennis. On the superlinear and quadratic convergence of primal-dual interior-point linear programming algorithms. SIAM J. on Optimization, 2:304$324,1992$. 